\title{
Intrathoracic tumors in contact with the chest wall - ultrasonographic and computed tomography guided biopsy comparative evaluation
}

\author{
Yaser G. Abish and Ahmad Okasha Mohammed \\ Department of Diagnostic Radiology, Al-Azhar Faculty of Medicine (Cairo and Asuit), Egypt.
}

\begin{abstract}
:
Purpose: To retrospectively compare the outcome of computed tomography (CT) and ultrasonography (US) guidance when sampling a consecutive series of peripheral lung or pleural lesions.

Materials And Methods: Institutional review board approval was obtained, and the informed consent requirement was waived. From March 2010 to July 2012, 550 thoracic biopsies were performed at Al Azher unveristy hostiptals (cairo and assuit)Damanhour Oncolgy Center. Among these, 260 lesions in 260 patients (110 men, 150 women; mean age, 66 years \pm 23 [standard deviation]; 81 pleural lesions; 179 pulmonary lesions) had pleural origin or were peripherally located in the lung with a small amount of pleural contact. These lesions were sampled with either CT (162 patients; mean age, 65 years $\pm 22 ; 52$ pleural lesions, 110 peripheral pulmonary lesions) or US (98 patients; mean age, 66 years \pm 16; 29 pleural lesions, 69 peripheral pulmonary lesions) guidance by using an 18-gauge modified Menghini needle. Procedure duration, postprocedural pneumothorax or hemorrhage, and sample adequacy were recorded. Fisher exact test, log-rank test, and Mann-Whitney U test were performed.

Results: No significant difference was found for patient age $(P=.741)$, sex $(P=.900)$, lesion size $(P=.206)$, or lesion origin $(P=.788)$. Median time was 576 seconds for $C T$-guided biopsy (25th percentile, 418 seconds; 75th percentile, 714 seconds) and 311 seconds for US-guided biopsy (25th percentile, 152 seconds; 75th percentile, 480 seconds $)(P<.001)$. Post-procedural pneumothorax was observed in 24 of 162 (14.8\%) CTguided procedures and in five of $98(5.1 \%)$ US-guided procedures $(P=.025)$; hemorrhage occurred in two of $162(1.2 \%) C T$-guided procedures and in one of $98(1.0 \%)$ US-guided procedures $(P=.875)$. Technical success was achieved in 95 of 98 US-guided procedures (97\%) and in 156 of 162 CT-guided procedures (96.3\%) $(P=$ .999).

Conclusion:US guidance is comparable to CT guidance in sampling pleural or peripheral lung lesions, in terms of sample accuracy, while allowing for a significant reduction in procedure time and post-procedural pneumothorax and being free from ionizing radiation.
\end{abstract}

Keywords: Intrathoracic tumors, Ultrasound, Computriazd tomography

Department of Diagnostic Radiology, Al-Azhar Faculty of Medicine (Asuit), Egypt.

Conflict of interest: None

Funding: This research did not receive any specific grant from any funding agency in the public, commercial or not-for-profit sector.

\section{Introduction}

Percutaneous biopsy yields tissue samples for diagnosis and staging and facilitates differentiation of primary cancer from distant metastasis or infective and inflammatory lesions, which is crucial for correct management of lung lesions (1). Percutaneous biopsy has also been used to obtain tissue for genetic and immunologic testing of cancer mutations, thus improving the care of patients undergoing targeted chemotherapy

Imaging -guided percutaneous trans-thoracic biopsy has become a widely accepted , effective, and safe minimally invasive technique with which to obtain tissue specimens from a number of different intra-thoracic lesions (4-6) .

Computed tomography (CT) is the most common guidance technique for percutaneous trans-thoracic interventional procedures, as it provides relevant information about needle trajectory within the lung $(4,7,8)$. However, conventional CT guidance does not allow for real-time monitoring of both needle movement and lesion displacement with respiration. To overcome these limits, CT fluoroscopy has been introduced recently. This tool allows for continuous monitoring of both lung movements and needle course as it progresses toward the lesion (9-11) however, both of these imaging modalities use a large amount of ionizing radiation and are capable of providing only axial sections of the lung in real time $(6,12)$.

In the chest, the role of ultrasonography (US) has traditionally been limited when compared with CT, since US waves are not able to penetrate aerated tissue (13). As a consequence, US has mainly been used to evaluate 
pleural effusion, pneumothorax, and pulmonary fibrosis and to serve as guidance for thoracentesis and chest tube placement (13-15). However, US has been described as a useful tool in the characterization of undetermined pleural or sub-pleural pulmonary nodules $(13,16)$ when no aerated lung is interposed in between . Also, US can be used as a safe and effective method with which to guide biopsies of masses abutting the chest wall $(5,17-20)$. Major advantages of this imaging modality are that US enables real time multiplanar monitoring

of the procedure and can be performed at the patient bedside, without the use of ionizing radiation . This study was conducted to compare the outcome of CT and US guidance when sampling peripheral pleuro-

\section{PATIENTS AND METHODS} pulmonary lesions .

\section{Patients:.}

Institutional review board approval was obtained, and the need for patient informed consent was waived. We retrospectively reviewed our case series of patients who underwent pulmonary biopsy with imaging guidance at Damanhour oncology center from January 2009 to March 2012. In this period 260 patients who had pleural or peripherally located lung lesions with at least a small amount of contact with the pleura entered our analysis .Demographics and data regarding anatomic position of nodules are reported in table 1.

\section{CT and US Guidance Technique}

All procedures were performed of without the use of a specific protocol. However patient group was homogenous for anatomic location of the lesion, which included both peripheral pulmonary and pleural lesions (Table 1)

CT guidance was performed by using 4-section multi-detector CT system (Toshiba Asteion 4 slice) . Patients were positioned on CT table in prone, supine, or lateral decubitus position depending on the location of the lesion, to obtain the most direct route for biopsy. The skin entry site was marked by using the laser light from the CT gantry. The skin was cleaned with $10 \%$ iodopovidine (or a mixture of $2 \%$ chlorexidine gluconate and $70 \%$ ethyl alcohol if the patient had an allergy to iodine). Local anesthetic (up to $10 \mathrm{~mL}$ of $2 \%$ mepivacaine hydrochloride without adrenaline) was injected up to the pleural surface . At this moment, needle position was checked By performing a CT scan. Thereafter, the anesthesia needle was removed and the biopsy needle was advanced through the thoracic wall and the pleura . CT images were used to confirm the correct placement of the biopsy needle within the lesion (fig1).

US guidance was performed by using a US system (Toshiba, Xario 200 ) equipped with a 3.5-5.0 MHz convex probe. Patient position was decided according to the best way to reach the lesion. The US probe was covered by a sterile condom to ensure complete sterilization after administration of local antiseptic and anesthetic, the biopsy needle was inserted by using a sterile puncture transducer with a needle -guide attachment with different angle selection or a freehand out-of-plane approach (fig2a\&b). the biopsy needle was then advanced into the lesion with real time visualization .

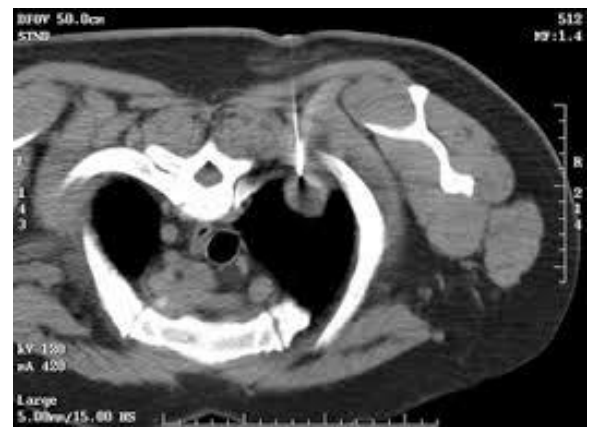

Figure 1: CT guided biopsy of posterior peripheral pulmonary lesion.

All sample were acquired by using an 18 -gauge modified Menghini needle (Length range 10-15 cm) and all specimens were placed in formaldehyde-filled sterile container . 


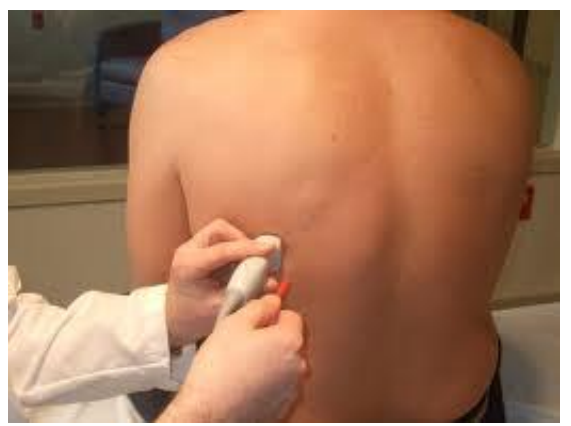

Figure 2a: US guided biopsy by using free hand out of plane approach.
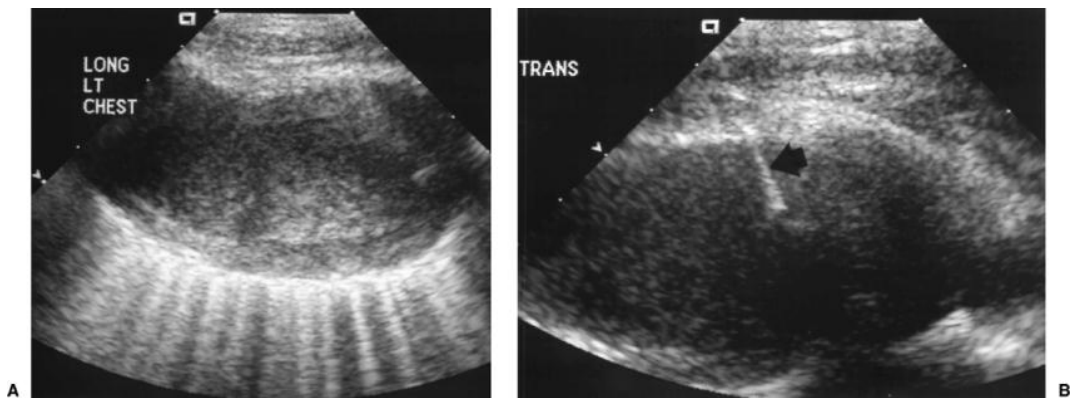

Figure 2b US guided biopsy of peripheral lung lesion (the needle is clearly seen (black arrow).

\section{Post-procedure Care}

After US-and CT- guided biopsy, patient were monitored for about 30 minutes after that a low -dose CT scan was performed through the whole chest to demonstrate the presence of post-procedural pneumothorax or intraparenchymal hemorrhage .

\section{Data Analysis}

Maximum diameter of lesions was recorded. Duration of both CT- and US- guided procedures was evaluated on the basis of picture archiving and communication system (PACS) storage time starting with the first image used to localize the lesion and ending with last image used as post-procedural control. Technical success evaluation and biopsy results were based on a review of histopathology records.

\section{Cost}

The cost of the procedure was calculated on the basis of global operational expenditures incurred by hospital for operators, use of the room, syringes, needles, and drugs. All these data were provided by the regional health administration.

\section{Statistical Analysis}

Age and maximum lesion diameter in patient who underwent CT-guided biopsy were compared with those of patient who underwent US-guided biopsy by using Mann -Whitney U test. Procedure duration for patient who underwent CT-guided biopsy was compared with that for patient who underwent US-guided biopsy by using the log-rank test, sex, lesion location (pleural or peripheral pulmonary) , technical success (sample adequacy for pathological diagnosis), and post-procedural pneumothorax rate for patient who underwent CT-guided biopsy were compared with those who underwent US-guided biopsy by using the Fisher exact test.

A P value less than .05 was considered indicative of a significant difference. SPSS software (version 17.0:IBM. Armonk, NY) was used.

\section{Results}

The target was reached successfully in all cases with both guidance techniques.

No significant difference was found between patient who underwent US-guided biopsy and patients who underwent CT-guided biopsy with regards to patient,s age, sex , lesion size, and lesion origin ( $\mathrm{P}>$.206). Detailed data are reported in Table 1 . 
Table 1 : Lesion Distribution And demographics in 260 pleural \&peripheral pulmonary lesion

\begin{tabular}{|r|r|r|r|r|}
\hline Parameter & Overall & US Guidance I & CT Guidance & P value \\
\hline $\begin{array}{r}\text { No. of patients } \\
\text { Men }\end{array}$ & $\mathbf{2 6 0}$ & $\mathbf{9 8}$ & $\mathbf{1 6 2}$ & \\
women & 110 & 41 & 68 & .900 \\
Mean age (y) & 150 & 57 & 94 & .900 \\
Men & $\mathbf{6 6} \pm \mathbf{2 3}$ & $\mathbf{6 6} \pm \mathbf{1 6}$ & $\mathbf{6 5} \pm \mathbf{2 2}$ & \\
women & $65 \pm 12$ & $67 \pm 25$ & $67 \pm 21$ & $\mathbf{7 4 1}$ \\
Pleural & $81(31.2 \%)$ & $29(29.6 \%)$ & $66 \pm 22$ & $\mathbf{7 4 1}$ \\
\hline No. of lesions: & & & $\mathbf{9 8}$ & \\
Peripheral & $179(68.8 \%)$ & $69(70.4 \%)$ & $110(67.9 \%)$ & .788 \\
pulmonary & & & & \\
\hline Mean lesion diameter & $\mathbf{2 . 5} \pm \mathbf{0 . 9}$ & $\mathbf{2 . 7} \pm \mathbf{0 . 9}$ & $\mathbf{2 . 3} \pm \mathbf{0 . 8}$ & $\mathbf{. 2 0 6}$ \\
(cm.) & & & & \\
\hline
\end{tabular}

Median procedure duration was significantly shorter $(\mathrm{P}<.001)$ in patients who underwent the US-guided procedure (median time, 311 seconds, $25^{\text {th }}$ and $75^{\text {th }}$ percentiles, 152 and 480 seconds, respectively) than in those who underwent the CT-guided procedure (median time, 576 seconds, $25^{\text {th }}$ and $75^{\text {th }}$ procedures, 418 and 714 seconds, respectively).

There were 24 of 162 (14.8\%) pneumothorax after CT guided procedure and five of $98(5.1 \%)$ pneumothoraxes after US-guided procedures ( $\mathrm{P}=.025$ ). The post-procedural pneumothoraxes was 29 of 260(11.2\%).

Intra-parenchymal hemorrhage occurred in three of $260(1.1 \%)$ procedures overall-in two of 162(1.2\%) procedures performed with CT guidance and in one of $98(1.0 \%)$ procedure performed with US guidance $(\mathrm{P}=875)$.

Technical success was achieved in 95 of 98 biopsies (97\%) performed with US guidance and in 156 of 1162 biopsies $(96.3 \%)$ performed with CT guidance $(\mathrm{p}=.999)$. Detailed data about biopsy results are reported in table 2.

Table 2: Distribution of biopsy results in 260 pleural \&peripheral pulmonary lesions

\begin{tabular}{|c|c|c|c|c|}
\hline Biopsy Results & \multicolumn{2}{|c|}{ No. of US guided biopsy } & \multicolumn{2}{|c|}{ No. of CT guided biopsy } \\
\hline Malignant & 70 & $71.4 \%$ & 111 & $68.5 \%$ \\
\hline Adeno-carcinoma & 44 & $44.9 \%$ & 58 & $35.8 \%$ \\
\hline Lymphoma & 4 & $4.0 \%$ & 6 & $3.7 \%$ \\
\hline Metastasis & 6 & $6.1 \%$ & 12 & $7.4 \%$ \\
\hline Mesothelioma & 8 & $8.2 \%$ & 13 & $8.0 \%$ \\
\hline Neuroendocrine Tumor & 1 & $1.0 \%$ & 3 & $1.9 \%$ \\
\hline Squamous carcinoma & 3 & $3.0 \%$ & 10 & $6.2 \%$ \\
\hline Undifferentiated malignancy & 4 & $4.0 \%$ & 9 & $5.6 \%$ \\
\hline Benign & 21 & $21.4 \%$ & 29 & $17.9 \%$ \\
\hline Negative & 4 & $4.0 \%$ & 16 & $9.9 \%$ \\
\hline Inadequate & 3 & $3.0 \%$ & 6 & $3.7 \%$ \\
\hline Total & 98 & & 162 & $100 \%$ \\
\hline
\end{tabular}

CT guided procedures had a comprehensive cost of about 430 LE for pleural or peripheral lung lesion, while US- guided procedures had a comprehensive cost of about $320 \mathrm{LE}$ for both pleural and peripheral lung lesions.

\section{Disccusion}

Our experience demonstrated that US guidance is as accurate as CT guidance in obtaining adequate samples from pleural and peripheral lung lesions. Also, in our series, the use of US guidance led to a significant reduction in mean procedural time and post procedural pneumothorax incidence when compared with $\mathrm{CT}$ 
guidance . CT provides excellent contrast and spatial resolution and enables accurate needle placement, making it the most widely used guidance technique for percutaneous trans-thoracic interventional procedures $(4,7,8)$. However, the use of ionizing radiation, the lack of real time capability, and the visualization of only transverse sections are the major limitations of CT guidance. This specially true with small lesions or those that are difficult to sample, where multiple sections acquisitions and needle re-positioning may be necessary. These complications can increase patient discomfort, as well as the length of time during which the needle is positioned across the pleura. The introduction of CT fluoroscopy has overcome the limitation of real-time needle visualization during CT- guided procedures, but it still provides only transverse sections and introduces a substantial amount of radiation exposure to both patient and operator(6). On the other hand, real time guidance can be performed by using US, making the procedure faster and free of ionizing radiation. In addition, US equipment is less expensive than CT systems and is generally more widely available. Portable US machines can also be moved to the patient,s bedside, so the procedure can be performed at the bedside of critically ill patients and those situated in different positions, such as semi-sitting decubitus position in patients with dyspnea who cannot tolerate prone or supine positioning .

In 84 consecutive patients who underwent percutaneous trans-thoracic biopsies, Sheth et al (20) were able to perform US guided biopsy in $40 \%$ of their patient, with a correct diagnosis in $91 \%$ of them. Moreover transthoracic US was demonstrated to be as effective as chest radiography in the detection of pneumothorax after trans-thoracic US-guided lung biopsy, thus allowing monitoring of post-procedural complication, as well (21). This last option we was not used in our series, as we always used a CT scan to demonstrate the presence of

post-procedural pneumothorax or hemorrhage .

Our results demonstrated that US-guided biopsy is as accurate as CT-guided biopsy in the diagnosis of peripheral pulmonary or pleural lesions. This is somewhat intuitive, as once the needle is placed correctly, ,sampling is performed with the same needle in the same way .

In our study, we found two main benefits of US guidance when performing lung biopsies, First ,US guidance allowed for a significantly (46\%) shorter procedure time, as needle placement and biopsy can be performed during one breath hold, this is particularly useful in small mobile basilar lesion, when even a slight variation in respiratory excursion can affect the position of the lesion, and in non-collaborative patients with dyspnea, in whom it is particularly difficult to obtain a prolonged and stable breath hold , together with reducing patient discomfort, this decreases the time the needle is placed across the pleura. Second we found a significantly lower incidence of post-procedural pneumothoraxes in patients who underwent US-guided procedures compared with those who underwent CT-guided biopsy. These data can be explained by the fact that real-time monitoring with US guidance enables a more straightforward puncture of the lesion, thus preventing the possible multiple punctures that are sometimes needed when sampling is performed with CT guidance. Also, a reduction of procedural time could be have positively conditioned the lower incidence of post-procedural pneumothoraxes in patients who underwent US-guided sampling. Sheth et al (20) needed 1884 seconds to perform US-guided thoracic biopsy. The value in our study are far lower than the values in their study. This can be explained by the fact that they considered the whole duration of the procedure, while our data merely are based on PACS storage timing, thus, our values represent an underestimation of the time needed to perform CT- or US-guided thoracic biopsy overall, but they can still be used for a reliable comparison between patients included in our series.

Pneumothorax is the most common complication of CT-guided trans-thoracic pulmonary biopsy with a reported frequency between $2.8 \%$ and $46 \%(6.13 .21 .22)$. Our overall value of $11.2 \%$ sits within the range . Similarly, intra-parenchymal hemorrhage is an uncommon event when dealing with pleural or peripheral lung lesions (20). In our series, we found three cases in which post-procedual CT showed a thin blood layer around the lesion.

Adequacy rate of peripheral pulmonary or pleural lesion biopsy performed with a non fine needle is reported to be between $89.5 \%$ and $97 \%$ with CT guidance $(6,9,10,23)$ and between $76 \%$ and $96 \%$ with US guidance $(17,19,20)$.

Our values are comparable with those reported in the literature.

Cost is another important factor to consider, if one assumes that no difference exist between CT and US guidance in the terms of safety and diagnostic accuracy, US-guided procedures less expensive than CT-guided procedures. We should highlight that the amount ionizing radiation administrated with a CT guided procedure can not be considered negligible.

In summary, when dealing with pleural or peripheral lung lesions, US guidance is comparable to CT in terms of sample accuracy, while allowing for a significant reduction in procedure time and post-procedural pneumothrax, without the use of ionizing radiation. 


\section{References}

[1]. Carrafiello G, Laganà D, Nosari AM, et al.. Utility of computed tomography (CT) and of fine needle aspiration biopsy (FNAB) in early diagnosis of fungal pulmonary infections: study of infections from filamentous fungi in haematologically immunodeficient patients. Radiol Med (Torino) 2006;111(1):33-41.

[2]. Cheung YC, Chang JW, Hsieh JJ, Lin G, Tsai YH. Adequacy and complications of computed tomography-guided core needle biopsy on non-small cell lung cancers for epidermal growth factor receptor mutations demonstration: 18-gauge or 20-gauge biopsy needle. Lung Cancer 2010;67(2):166-169.

[3]. Solomon SB, Zakowski MF, Pao W, et al.. Core needle lung biopsy specimens: adequacy for EGFR and KRAS mutational analysis. AJR Am J Roentgenol 2010;194(1):266-269.

[4]. Gupta S, Madoff DC. Image-guided percutaneous needle biopsy in cancer diagnosis and staging. Tech Vasc Interv Radiol 2007;10(2):88-101.

[5]. Schubert P, Wright CA, Louw M, et al.. Ultrasound-assisted transthoracic biopsy: cells or sections? Diagn Cytopathol 2005;33(4):233-237.

[6]. Kim GR, Hur J, Lee SM, et al.. CT fluoroscopy-guided lung biopsy versus conventional CT-guided lung biopsy: a prospective controlled study to assess radiation doses and diagnostic performance. Eur Radiol 2011;21(2):232-239.

[7]. Tsai IC, Tsai WL, Chen MC, et al.. CT-guided core biopsy of lung lesions: a primer. AJR Am J Roentgenol 2009;193(5):12281235 .

[8]. Rossi UG, Seitun S, Ferro C. MDCT-guided transthoracic needle aspiration biopsy of the lung using the transscapular approach. Cardiovasc Intervent Radiol 2011;34(1):184-187.

[9]. Heck SL, Blom P, Berstad A. Accuracy and complications in computed tomography fluoroscopy-guided needle biopsies of lung masses. Eur Radiol 2006;16(6):1387-1392.

[10]. Hiraki T, Mimura H, Gobara H, et al.. CT fluoroscopy-guided biopsy of 1,000 pulmonary lesions performed with 20-gauge coaxial cutting needles: diagnostic yield and risk factors for diagnostic failure. Chest 2009;136(6):1612-1617.

[11]. Carlson SK, Felmlee JP, Bender CE, et al.. CT fluoroscopy-guided biopsy of the lung or upper abdomen with a breath-hold monitoring and feedback system: a prospective randomized controlled clinical trial. Radiology 2005;237(2):701-708

[12]. Yamao Y, Yamakado K, Takaki H, et al.. Optimal scan parameters for CT fluoroscopy in lung interventional radiologic procedures: relationship between radiation dose and image quality. Radiology 2010;255(1):233-241.

[13]. Sartori S, Tombesi P, Trevisani L, Nielsen I, Tassinari D, Abbasciano V. Accuracy of transthoracic sonography in detection of pneumothorax after sonographically guided lung biopsy: prospective comparison with chest radiography. AJR Am J Roentgenol 2007;188(1):37-41.

[14]. Josephson T, Nordenskjold CA, Larsson J, Rosenberg LU, Kaijser M. Amount drained at ultrasound-guided thoracentesis and risk of pneumothorax. Acta Radiol 2009;50(1):42-47.

[15]. Chung MJ, Goo JM, Im JG, Cho JM, Cho SB, Kim SJ. Value of high-resolution ultrasound in detecting a pneumothorax. Eur Radiol 2005;15(5):930-935.

[16]. Rednic N, Orasan O. Subpleural lung tumors ultrasonography. Med Ultrasound 2010;12(1):81-87.

[17]. Diacon AH, Schuurmans MM, Theron J, Schubert PT, Wright CA, Bolliger CT. Safety and yield of ultrasound-assisted transthoracic biopsy performed by pulmonologists. Respiration 2004;71(5):519-522.

[18]. Ikezoe J, Morimoto S, Arisawa J, Takashima S, Kozuka T, Nakahara K. Percutaneous biopsy of thoracic lesions: value of sonography for needle guidance. AJR Am J Roentgenol 1990;154(6):1181-1185.

[19]. Liao WY, Chen MZ, Chang YL, et al.. US-guided transthoracic cutting biopsy for peripheral thoracic lesions less than $3 \mathrm{~cm}$ in diameter. Radiology 2000;217(3):685-691.

[20]. Sheth S, Hamper UM, Stanley DB, Wheeler JH, Smith PA. US guidance for thoracic biopsy: a valuable alternative to CT. Radiology 1999;210(3):721-726.

[21]. Sartori S, Tombesi P. Emerging roles for transthoracic ultrasonography in pleuropulmonary pathology. World J Radiol 2010;2(2):83-90.

[22]. Rizzo S, Preda L, Raimondi S, et al.. Risk factors for complications of CT-guided lung biopsies. Radiol Med (Torino) 2011;116(4):548-563.

[23]. Ohno Y, Hatabu H, Takenaka D, Imai M, Ohbayashi C, Sugimura K. Transthoracic CT-guided biopsy with multiplanar reconstruction image improves diagnostic accuracy of solitary pulmonary nodules. Eur J Radiol 2004;51(2):160-168. 